\title{
POINTWISE ERROR ESTIMATES FOR RELAXATION APPROXIMATIONS TO CONSERVATION LAWS*
}

\author{
EITAN TADMOR ${ }^{\dagger}$ AND TAO TANG TA $^{\ddagger}$
}

\begin{abstract}
We obtain sharp pointwise error estimates for relaxation approximation to scalar conservation laws with piecewise smooth solutions. We first prove that the first-order partial derivatives for the perturbation solutions are uniformly upper bounded (the so-called $\mathrm{Lip}^{+}$stability). A one-sided interpolation inequality between classical $L^{1}$ error estimates and Lip $^{+}$stability bounds enables us to convert a global $L^{1}$ result into a (nonoptimal) local estimate. Optimal error bounds on the weighted error then follow from the maximum principle for weakly coupled hyperbolic systems. The main difficulties in obtaining the $\mathrm{Lip}^{+}$stability and the optimal pointwise errors are how to construct appropriate "difference functions" so that the maximum principle can be applied.
\end{abstract}

Key words. conservation laws, error estimates, relaxation method, optimal convergence rate, one-sided interpolation inequality, maximum principle

AMS subject classifications. 35L65, 34C26, 65M10, 65M15

PII. S0036141098349492

1. Introduction. Consider the following stiff relaxation system:

$$
\left\{\begin{array}{l}
u_{t}^{\epsilon}+v_{x}^{\epsilon}=0 \\
v_{t}^{\epsilon}+\alpha u_{x}^{\epsilon}=-\frac{1}{\epsilon}\left(v^{\epsilon}-f\left(u^{\epsilon}\right)\right), \quad \epsilon>0
\end{array}\right.
$$

for $(x, t) \in \mathbf{R} \times(0, \infty)$. The initial conditions associated with the above system are

$$
u^{\epsilon}(x, 0)=u_{0}(x), \quad v^{\epsilon}(x, 0)=f\left(u_{0}(x)\right) .
$$

The system (1.1) can be regarded as a singular perturbation problem, and the solutions are expected to converge, as $\epsilon$ tends to zero, to the entropy solutions of the equilibrium equation

$$
\begin{cases}u_{t}+f(u)_{x}=0, & v=f(u), \\ u(x, 0)=u_{0}(x), & v(x, 0)=f\left(u_{0}(x)\right) .\end{cases}
$$

The relaxation limit for $2 \times 2$ nonlinear systems of conservation laws was first studied by Liu [11], who justified some nonlinear stability criteria for diffusion waves, expansion waves, and traveling waves. A general mathematical framework was analyzed for the nonlinear systems by Chen, Levermore, and Liu [1]. Consult [12] for a bird's eye view of recent results in this direction.

The presence of relaxation mechanisms is widespread in both the continuum mechanics as well as the kinetic theory contexts. Relaxation is known to provide a subtle

\footnotetext{
${ }^{*}$ Received by the editors December 21, 1998; accepted for publication (in revised form) August 23, 2000; published electronically December 13, 2000. This research was supported in part by ONR grant N00014-91-J-1076, NSF grant DMS97-06827, and NSERC Canada grant OGP0105545. Part of this research was carried out while the second author was visiting UCLA.

http://www.siam.org/journals/sima/32-4/34949.html

†Department of Mathematics, UCLA, Los Angeles, CA 90095 (tadmor@math.ucla.edu).

${ }^{\ddagger}$ Department of Mathematics, The Hong Kong Baptist University, Kowloon Tong, Hong Kong (tangtao@fisher.math.hkbu.edu.hk).
} 
dissipative mechanism for discontinuities against the destabilizing effect of nonlinear response [11]. The relaxation models can be loosely interpreted as discrete velocity kinetic equations. The relaxation parameter, $\epsilon$, plays the role of the mean free path and the system models the macroscopic conservation law. In that sense they are a discrete velocity analogue of the kinetic equations introduced by Perthame and Tadmor [15] and Lions, Perthame, and Tadmor [10].

The relaxation approximation can also be used to construct numerical approximations to the equilibrium conservation laws. In [6], Jin and Xin developed a class of first- and second-order nonoscillatory numerical schemes for the conservation law (1.3), based on the relaxation approximation (1.1). Since the relaxation approximation (1.1) is formally an $\mathcal{O}(\epsilon)$ perturbation to (1.3), they can compute (1.1) without resolving the computational grid to $\mathcal{O}(\epsilon)$. Indeed, in their final form, it is seen that the relaxation parameter in these relaxation schemes plays no role. In particular, their $\epsilon=0$-limit in the first-order case coincides with the central Lax-Friedrichs scheme, and their $\epsilon=0$-limit in the second-order version corresponds to the central scheme of Nessyahu and Tadmor [13]. The nonoscillatory central schemes introduced in [13] are based on staggered evolution of the reconstructed averages - a high-order sequel to the celebrated first-order Lax-Friedrichs (staggered) scheme. An extension of the high-resolution central scheme to multidimensional problems can be found in [5]. The central schemes are simple, efficient, stable, and enjoy the main advantage of avoiding costly (upwind) Riemann solvers. In this context, relaxation schemes offer yet another way to derive a whole class of high-resolution Riemann-solvers-free central schemes. The key is how to discretize the relaxation, as outlined in [6].

There have been many recent studies concerning the asymptotic convergence of the relaxation systems to the corresponding equilibrium conservation laws as the rate of the relaxation tends to zero. Most of these results deal with either large-time, nonlinear asymptotic stability or the zero relaxation limit for Cauchy problems. Tveito and Winther $[18,26]$ provided an $\mathcal{O}\left(\epsilon^{1 / 3}\right)$-rate of convergence for some relaxation systems with nonlinear convection arising in chromatography. Katsoulakis and Tzavaras [7] introduced a class of relaxation systems, the contractive relaxation systems, and established an $\mathcal{O}(\sqrt{\epsilon})$ error bound in the case that the equilibrium equation is a scalar multidimensional one. The approaches in $[7,18,26]$ are based on the extensions of Kruzhkov and Kuznetzov-type error estimates [9]. Kurganov and Tadmor [8] studied convergence and error estimates for a class of relaxation systems, including (1.1) and the one arising in chromatography, and concluded an $\mathcal{O}(\epsilon)$ order of convergence for scalar convex conservation laws. The novelty of their approach is the use of a weak Lip'-measure of the error, which allows them to obtain sharp error estimates. ${ }^{1}$ For the relaxation system (1.1), Natalini [12] proved that the solutions to the relaxation system converges strongly to the unique entropy solution of (1.2) as $\epsilon \rightarrow 0$. Based on a general framework developed in [23, 25], the first-order rate of convergence for (1.1) is established in the case when its equilibrium solutions are piecewise smooth [24], which is an improvement on the $\mathcal{O}(\sqrt{\epsilon})$ error bounds [7, 8]. The boundary layer effect in the small relaxation limit to the equilibrium scalar conservation laws was investigated in [27]. The existence and uniqueness for the initial-boundary value problems are established.

The convergence and the rate of convergence mentioned above are mostly in the $L^{1}$ sense. It is understood that the $L^{1}$ error estimate is a global one, while in many practical cases we are interested in the local behavior of $u(x, t)$. Consequently, when

\footnotetext{
${ }^{1}$ Here and below, Lip ${ }^{\prime}$ stands for the dual of Lip topology.
} 
the error is measured by the $L^{1}$-norm, there is a loss of information due to the poor resolution of shock waves in $u(x, t)$. Several authors have investigated pointwise error estimates: For a system of conservation laws, Goodman and Xin [4] proved that the viscosity methods approximating piecewise smooth solutions with finitely many noninteracting shocks have a local $\mathcal{O}(\epsilon)$ error bound away from the shocks. A general convergence theory for one dimensional (1D) scalar convex conservation laws was developed by Tadmor and coauthors; see, e.g., [13, 19]. They proved that when measured in the weak Lip'-topology, the convergence rate of the viscous solution is of order $\mathcal{O}(\epsilon)$ in the case of rarefaction-free initial data and is of order $\mathcal{O}(\epsilon|\ln \epsilon|)$ in the general case. These weak $L i p^{\prime}$-estimates are then converted into the usual $L^{1}$ error bounds of order one-half, and moreover, pointwise error estimates of order one-third, $\mathcal{O}\left(\epsilon^{1 / 3}\right)$, are derived. Pointwise error analysis for finite difference methods to scalar and system of conservation laws is given recently by Engquist and $\mathrm{Yu}$ [3], Engquist and Sjogreen [2]. In [20], the authors provided the optimal pointwise convergence rate for the viscosity approximation. They used an innovative idea which enables them to convert a global $L^{1}$-error estimate into a local error estimate. Using this local error estimate and a bootstrap argument they proved that the viscosity approximation satisfies a pointwise error estimate of order $\mathcal{O}(\epsilon)$ for all but finitely many neighborhoods of shock discontinuities, each of width $\mathcal{O}(\epsilon)$. The previous results for the optimal order one convergence rates, in both $L^{1}$ and $L^{\infty}$ spaces, are all based on a matching method and traveling wave solutions; see, e.g., [3, 4, 23]. The approach introduced in [20] does not follow the characteristics but instead makes use of the energy method, and hence can be extended to other types of approximate solutions, e.g., [21].

The question that we address in this paper is concerned with the rate of pointwise convergence for the relaxation approximation (1.1). The main purpose is to establish the optimal pointwise convergence. The proof of our results is based on two ingredients:

- a one-sided interpolation inequality between the $L^{1}$ error estimates and Lip $^{+}$ stability bounds; and

- a comparison theorem (the maximum principle) for weakly coupled hyperbolic systems.

In section 2, we review the preliminary results required for obtaining our error bounds. As mentioned earlier, the $L^{1}$ error bounds for the relaxation approximation have been established by several authors. A rigorous $\mathrm{Lip}^{+}$stability bounds for the relaxation approximation will be established in section 3 . In section 4 we first consider the case when there is only one shock in the solutions of the equilibrium equation (1.3); i.e., the set of shock $S$ consists of only one smooth curve. In this case, we show that

$$
\operatorname{dist}(x, S)\left|u(x, t)-u^{\epsilon}(x, t)\right| \leq C \epsilon .
$$

It implies that $\left|u(x, t)-u^{\epsilon}(x, t)\right| \leq C(h) \epsilon$ for $(x, t)$ which are at least $\mathcal{O}(h)$ away from the set of shocks. The result (1.4) can be generalized to finitely many shocks with possible collisions. In the final section, we discuss the possible extensions of the results obtained in this work.

2. Preliminaries. Several useful results for the relaxation approximation will be reviewed in this section. We begin by introducing the subcharacteristic condition.

2.1. Subcharacteristic condition. The main stability criterion can be (formally) derived by using the Chapman-Enskog expansion for the stiff relaxation 
system (1.1)

$$
u_{t}^{\epsilon}+f\left(u^{\epsilon}\right)_{x}=\epsilon\left(\left(\alpha-f^{\prime}\left(u^{\epsilon}\right)^{2}\right) u_{x}^{\epsilon}\right)_{x}+\mathcal{O}\left(\epsilon^{2}\right)
$$

The above equation will be of parabolic type under the following stability condition, i.e., the subcharacteristic condition [28]:

$$
\alpha>f^{\prime}\left(u^{\epsilon}\right)^{2} .
$$

In a recent paper, Natalini [12] provided a rigorous analysis for (1.1) that leads to the subcharacteristic condition (2.2) under some assumptions on $\alpha$ and the initial data $u_{0}$. More precisely, we state his results as follows.

Lemma 2.1. If $\alpha$ in the relaxation equation (1.1) and the initial data $u_{0}$ in (1.2) satisfy

$$
\sqrt{\alpha}>M\left(N_{0}\right),
$$

where $N_{0}$ and $M_{0}$ are defined by

$$
\left\{\begin{array}{l}
N_{0}:=\max \left(\left\|u_{0}\right\|_{L^{\infty}},\left\|f\left(u_{0}\right)\right\|_{L^{\infty}}\right), M\left(N_{0}\right):=\sup _{|\zeta| \leq B\left(N_{0}\right)}\left|f^{\prime}(\zeta)\right|, \text { with } \\
B\left(N_{0}\right):=2 N_{0}+F\left(2 N_{0}\right), F\left(N_{0}\right):=\sup _{|\zeta| \leq N_{0}}|f(\zeta)|
\end{array}\right.
$$

then the relaxation system (1.1) with initial condition (1.2) satisfies the subcharacteristic inequality (2.2). Moreover, the solution $\left(u^{\epsilon}, v^{\epsilon}\right)$ for (1.1) is uniformly bounded with respect to $\epsilon$ :

$$
\left|u^{\epsilon}(x, t)\right| \leq B\left(N_{0}\right),\left|v^{\epsilon}(x, t)\right| \leq \sqrt{\alpha} B\left(N_{0}\right), \text { for }(x, t) \in \mathbf{R} \times(0, \infty) .
$$

Throughout this paper, we will assume that the condition (2.3) is satisfied. Under this assumption, the subcharacteristic inequality is guaranteed and will be used to establish the $\mathrm{Lip}^{+}$stability and the pointwise error bounds.

2.2. Global $L^{1}$ error bounds. The $L^{1}$-error analysis for the relaxation approximation method has been presented by several authors. For general data, an optimal $L^{1}$-rate can be found in [7,8], for example. This optimal $\mathcal{O}(\sqrt{\epsilon}) L^{1}$-rate is overviewed in section 3.2, based on the $\mathrm{Lip}^{\prime}$ approach taken in [8] (for a more general class of relaxation models). For piecewise-smooth data, the optimal $\sim \mathcal{O}(\epsilon) L^{1}$-convergence rate was recently obtained by Teng [24]. We state his results as follows.

Lemma 2.2. Assume $\alpha$ in the relaxation equation (1.1) and the initial data $u_{0}$ in (1.2) satisfy the conditions stated in Lemma 2.1. Assume that the solutions to the scalar convex conservation law (1.3) are piecewise smooth. Let $\left(u^{\epsilon}, v^{\epsilon}\right)$ be the solutions of the relaxation problems (1.1)-(1.2). Then the following error estimate holds:

$$
\sup _{0 \leq t \leq T}\left(\left\|u^{\epsilon}(\cdot, t)-u(\cdot, t)\right\|_{L^{1}(\mathbf{R})}+\left\|v^{\epsilon}(\cdot, t)-v(\cdot, t)\right\|_{L^{1}(\mathbf{R})}\right) \leq C(T) \epsilon|\ln \epsilon|,
$$

where $v=f(u)$. If there is no initial central rarefaction wave and no new generated shocks, then the error bound is improved to

$$
\sup _{0 \leq t \leq T}\left(\left\|u^{\epsilon}(\cdot, t)-u(\cdot, t)\right\|_{L^{1}(\mathbf{R})}+\left\|v^{\epsilon}(\cdot, t)-v(\cdot, t)\right\|_{L^{1}(\mathbf{R})}\right) \leq C(T) \epsilon .
$$


We shall utilize these $L^{1}$ global error bounds to derive the pointwise error estimate (1.4). The order of the global $L^{1}$ error bounds will not affect the general $\mathcal{O}(\epsilon)$ pointwise result (1.4), but it will affect the choice of the distance function - see [20] for details. Thus improved $L^{1}$-error bounds lead to sharper description of the shock layer, with an optimal shock layer of size $\sim \mathcal{O}(\epsilon)$ corresponding to the piecewisesmooth cases (2.6) and (2.7).

2.3. An interpolation inequality. We let $\|\bullet\|_{L i p^{+}}$denote the Lip $^{+}$-seminorm

$$
\|w\|_{L_{i p^{+}}}:=\operatorname{ess} \sup _{x \neq y}\left[\frac{w(x)-w(y)}{x-y}\right]^{+},
$$

where $[w]^{+}=H(w) w$, with $H(\bullet)$ the Heaviside function. The following lemma is due to Nessyahu and Tadmor [14, section 2]; its proof can be found in [20].

Lemma 2.3. Assume that $z \in L^{1} \cap \operatorname{Lip}^{+}(\mathcal{I})$, and $w \in C_{l o c}^{1}(x-\delta, x+\delta)$ for an interior $x$ such that $(x-\delta, x+\delta) \subset \mathcal{I}$. Then the following estimate holds:

$$
|z(x)-w(x)| \leq \text { Const } \cdot\left[\frac{1}{\delta}\|z-w\|_{L^{1}}+\delta\left\{\|z\|_{L i p^{+}(x-\delta, x+\delta)}+|w|_{C_{l o c}^{1}(x-\delta, x+\delta)}\right\}\right] .
$$

In particular, if the size of the smoothness neighborhood for $w$ can be chosen so that

$$
\delta \sim\|z-w\|_{L^{1}(\mathcal{I})}^{1 / 2} \cdot\left(\|z\|_{L_{i p^{+}}}+|w|_{C_{l o c}^{1}}\right)^{-1 / 2} \leq \frac{1}{2}|\mathcal{I}|,
$$

then the following estimate holds:

$$
|z(x)-w(x)| \leq \text { Const } \cdot\|z-w\|_{L^{1}(\mathcal{I})}^{1 / 2} \cdot\left[\|z\|_{L i p^{+}}+|w|_{C_{l o c}^{1}(x-\delta, x+\delta)}\right]^{1 / 2}
$$

Thus (2.10) tells us that if the global $L^{1}$-error $\|z-w\|_{L^{1}}$ is small, then the pointwise error $|z(x)-w(x)|$ is also small wherever $w_{x}$ is bounded. This does not require the $C^{1}$-boundedness of $z$ - the weaker one-sided Lip $^{+}$bound of $z$ will suffice.

2.4. A comparison lemma. The following maximum principle for weakly coupled hyperbolic systems plays an important role in this work. Consider the following system:

$$
\left\{\begin{array}{l}
\partial_{t} u_{1}+\lambda_{1}(x, t) \partial_{x} u_{1}=\alpha_{11}(x, t) u_{1}+\alpha_{12}(x, t) u_{2}+\beta_{1}(x, t), \\
\partial_{t} u_{2}+\lambda_{2}(x, t) \partial_{x} u_{2}=\alpha_{21}(x, t) u_{1}+\alpha_{22}(x, t) u_{2}+\beta_{2}(x, t),
\end{array}\right.
$$

with $C^{1}$ local speeds, $\lambda_{i}(\cdot)$, and low-order terms on the right involving bounded coefficients, $\alpha_{i j}(\cdot), \beta_{i}(\cdot), 1 \leq i, j \leq 2$. The following lemma (see, e.g., [16, Theorem 13]), provides sufficient conditions which guarantee that if the initial and boundary data prescribed for (2.11) is nonpositive, the solution remains nonpositive.

Lemma 2.4. Consider the Cauchy problem for the weakly coupled hyperbolic systems (2.11) in a domain $E:=\mathbf{D} \times(0, T)$, subject to nonpositive initial and boundary conditions

$$
\begin{aligned}
& u_{1}(x, 0) \leq 0, \quad u_{2}(x, 0) \leq 0, \quad x \in \mathbf{D}, \\
& u_{1}(x, t) \leq 0, \quad u_{2}(x, t) \leq 0, \quad(x, t) \in \partial \mathbf{D} \times(0, T) .
\end{aligned}
$$


Assume that the coefficient functions in (2.11) satisfy

$$
\begin{aligned}
\alpha_{12}(x, t) \geq 0, \quad \alpha_{21}(x, t) & \geq 0,(x, t) \in E, \\
\beta_{1}(x, t) \leq 0, \quad \beta_{2}(x, t) & \leq 0,(x, t) \in E .
\end{aligned}
$$

Then the solution of (2.11) remains nonpositive in later time:

$$
u_{1}(x, t) \leq 0, \quad u_{2}(x, t) \leq 0 \quad \text { for } \quad(x, t) \in E .
$$

For the proof, we note that thanks to (2.13), the nonpositive maximal values, $U_{i}(t):=\sup _{x} u_{i}(x, t)$ are majorized by the ODEs, $\dot{U}_{i}=\alpha_{i i} U_{i}(t)+\alpha_{i j} u_{j}(t)+\beta_{i}(t) \leq$ $\alpha_{i i} U_{i}(t)$, and hence these maximal values cannot increase in time.

The two important results, the $\mathrm{Lip}^{+}$stability and the optimal pointwise error bounds are all based on the above lemma. The main difficulty is how to construct appropriate object functions $u_{1}, u_{2}$ so that above lemma can be suitably applied.

3. Lip $^{+}$stability and local error bounds. In this section, we assume that $f$ is strictly convex, i.e.,

$$
f^{\prime \prime}(u) \geq \beta>0 \quad \text { for } \quad u \in \mathbf{R},
$$

and that $u_{0}$ is Lip $^{+}$-bounded,

$$
\left\|u_{0}\right\|_{L i p^{+}}<\infty
$$

Definition 3.1. We say that $\left\{u^{\epsilon}(x, t)\right\}_{\epsilon>0}$ are Lip ${ }^{+}$-stable if the following estimate is fulfilled:

$$
\left\|u^{\epsilon}(\cdot, t)\right\|_{L i p^{+}} \leq\left\|u_{0}\right\|_{L i p^{+}}, \quad t \geq 0 .
$$

3.1. Lip $^{+}$stability. We will show that the family $\left\{u^{\epsilon}(x, t)\right\}_{\epsilon>0}$ is $\mathrm{Lip}^{+}$-stable. Assume first that $u_{0} \in C_{0}^{1}(\mathbf{R})$. This implies, by the standard regularity theory for the semilinear hyperbolic problems, that $\left(u^{\epsilon}, v^{\epsilon}\right) \in C^{1}(\mathbf{R} \times(0, T))$ for some $T>0$. Differentiating the equations (1.1) with respect to $x$ gives

$$
\begin{aligned}
\left(u_{x}^{\epsilon}\right)_{t}+\left(v_{x}^{\epsilon}\right)_{x} & =0 \\
\left(v_{x}^{\epsilon}\right)_{t}+\alpha\left(u_{x}^{\epsilon}\right)_{x} & =-\frac{1}{\epsilon}\left(v_{x}^{\epsilon}-f^{\prime}\left(u^{\epsilon}\right) u_{x}^{\epsilon}\right) .
\end{aligned}
$$

By doing $\sqrt{\alpha} \times(3.4)+(3.5)$ and $\sqrt{\alpha} \times(3.4)-(3.5)$, the above system can be put in the following diagonal form:

$$
\begin{aligned}
& \left(\sqrt{\alpha} u_{x}^{\epsilon}+v_{x}^{\epsilon}\right)_{t}+\sqrt{\alpha}\left(\sqrt{\alpha} u_{x}^{\epsilon}+v_{x}^{\epsilon}\right)_{x}=-\frac{1}{\epsilon}\left(v_{x}^{\epsilon}-f^{\prime}\left(u^{\epsilon}\right) u_{x}^{\epsilon}\right), \\
& \left(\sqrt{\alpha} u_{x}^{\epsilon}-v_{x}^{\epsilon}\right)_{t}-\sqrt{\alpha}\left(\sqrt{\alpha} u_{x}^{\epsilon}-v_{x}^{\epsilon}\right)_{x}=\frac{1}{\epsilon}\left(v_{x}^{\epsilon}-f^{\prime}\left(u^{\epsilon}\right) u_{x}^{\epsilon}\right) .
\end{aligned}
$$

Letting

$$
\bar{p}=\sqrt{\alpha} u_{x}^{\epsilon}+v_{x}^{\epsilon}, \quad \bar{q}=\sqrt{\alpha} u_{x}^{\epsilon}-v_{x}^{\epsilon}
$$

and by using the above results yield

$$
\left\{\begin{array}{l}
\bar{p}_{t}+\sqrt{\alpha} \bar{p}_{x}=\frac{1}{2 \epsilon}\left(\frac{f^{\prime}\left(u^{\epsilon}\right)}{\sqrt{\alpha}}-1\right) \bar{p}+\frac{1}{2 \epsilon}\left(\frac{f^{\prime}\left(u^{\epsilon}\right)}{\sqrt{\alpha}}+1\right) \bar{q} \\
\bar{q}_{t}-\sqrt{\alpha} \bar{q}_{x}=-\frac{1}{2 \epsilon}\left(\frac{f^{\prime}\left(u^{\epsilon}\right)}{\sqrt{\alpha}}-1\right) \bar{p}-\frac{1}{2 \epsilon}\left(\frac{f^{\prime}\left(u^{\epsilon}\right)}{\sqrt{\alpha}}+1\right) \bar{q}
\end{array}\right.
$$


We further introduce the transformations

$$
\left\{\begin{array}{l}
p=\bar{p}-\left(\sqrt{\alpha}+f^{\prime}\left(u^{\epsilon}\right)\right)\left\|u_{0}\right\|_{L i p^{+}}, \\
q=\bar{q}-\left(\sqrt{\alpha}-f^{\prime}\left(u^{\epsilon}\right)\right)\left\|u_{0}\right\|_{L i p^{+}} .
\end{array}\right.
$$

Applying the above transformations to (3.6) gives

$$
\begin{aligned}
p_{t}+\sqrt{\alpha} p_{x}= & \frac{1}{2 \epsilon}\left(\frac{f^{\prime}\left(u^{\epsilon}\right)}{\sqrt{\alpha}}-1\right) p+\frac{1}{2 \epsilon}\left(\frac{f^{\prime}\left(u^{\epsilon}\right)}{\sqrt{\alpha}}+1\right) q \\
& -f^{\prime \prime}\left(u^{\epsilon}\right)\left(u_{t}^{\epsilon}+\sqrt{\alpha} u_{x}^{\epsilon}\right)\left\|u_{0}\right\|_{L i p^{+}} \\
q_{t}-\sqrt{\alpha} q_{x}= & -\frac{1}{2 \epsilon}\left(\frac{f^{\prime}\left(u^{\epsilon}\right)}{\sqrt{\alpha}}-1\right) p-\frac{1}{2 \epsilon}\left(\frac{f^{\prime}\left(u^{\epsilon}\right)}{\sqrt{\alpha}}+1\right) q \\
& +f^{\prime \prime}\left(u^{\epsilon}\right)\left(u_{t}^{\epsilon}-\sqrt{\alpha} u_{x}^{\epsilon}\right)\left\|u_{0}\right\|_{L i p^{+}}
\end{aligned}
$$

It follows from (1.1), $u_{t}^{\epsilon}+v_{x}^{\epsilon}=0$, that

$$
\begin{gathered}
u_{t}^{\epsilon}+\sqrt{\alpha} u_{x}^{\epsilon}=-v_{x}^{\epsilon}+\sqrt{\alpha} u_{x}^{\epsilon}=q+\left(\sqrt{\alpha}-f^{\prime}\left(u^{\epsilon}\right)\right)\left\|u_{0}\right\|_{L i p^{+}}, \\
u_{t}^{\epsilon}-\sqrt{\alpha} u_{x}^{\epsilon}=-v_{x}^{\epsilon}-\sqrt{\alpha} u_{x}^{\epsilon}=-p-\left(\sqrt{\alpha}+f^{\prime}\left(u^{\epsilon}\right)\right)\left\|u_{0}\right\|_{L i p^{+}} .
\end{gathered}
$$

The above observation, together with (3.8) and (3.9), lead to

$$
\begin{aligned}
p_{t}+\sqrt{\alpha} p_{x}= & \frac{1}{2 \epsilon}\left(\frac{f^{\prime}\left(u^{\epsilon}\right)}{\sqrt{\alpha}}-1\right) p+\frac{1}{2 \epsilon}\left(\frac{f^{\prime}\left(u^{\epsilon}\right)}{\sqrt{\alpha}}+1-2 \epsilon f^{\prime \prime}\left(u^{\epsilon}\right)\left\|u_{0}\right\|_{L i p^{+}}\right) q \\
& -f^{\prime \prime}\left(u^{\epsilon}\right)\left(\sqrt{\alpha}-f^{\prime}\left(u^{\epsilon}\right)\right)\left\|u_{0}\right\|_{L i p^{+}}^{2} \\
q_{t}-\sqrt{\alpha} q_{x}= & -\frac{1}{2 \epsilon}\left(\frac{f^{\prime}\left(u^{\epsilon}\right)}{\sqrt{\alpha}}-1+2 \epsilon f^{\prime \prime}\left(u^{\epsilon}\right)\left\|u_{0}\right\|_{L i p^{+}}\right) p-\frac{1}{2 \epsilon}\left(\frac{f^{\prime}\left(u^{\epsilon}\right)}{\sqrt{\alpha}}+1\right) q \\
& -f^{\prime \prime}\left(u^{\epsilon}\right)\left(\sqrt{\alpha}+f^{\prime}\left(u^{\epsilon}\right)\right)\left\|u_{0}\right\|_{L i p^{+}}^{2} .
\end{aligned}
$$

It follows from the subcharacteristic condition (2.2) that (3.10)-(3.11) is a weakly coupled hyperbolic system and its coefficients satisfy the requirements in (2.12) provided that $\epsilon$ is sufficiently small. We now check the initial conditions. First checking $p(x, 0)$,

$$
\begin{aligned}
p(x, 0) & =\sqrt{\alpha} u_{0}^{\prime}+f^{\prime}\left(u_{0}\right) u_{0}^{\prime}-\left(\sqrt{\alpha}+f^{\prime}\left(u_{0}\right)\right)\left\|u_{0}\right\|_{L i p^{+}} \\
& =\left(\sqrt{\alpha}+f^{\prime}\left(u_{0}\right)\right)\left(u_{0}^{\prime}-\left\|u_{0}\right\|_{L i p^{+}}\right) \leq 0 .
\end{aligned}
$$

Similarly, we have

$$
q(x, 0)=\left(\sqrt{\alpha}-f^{\prime}\left(u_{0}\right)\right)\left(u_{0}^{\prime}-\left\|u_{0}\right\|_{L i p^{+}}\right) \leq 0 .
$$

Using Lemma 2.4, we obtain

$$
p(x, t) \leq 0, \quad q(x, t) \leq 0 \quad \text { for } \quad(x, t) \in \mathbf{R} \times(0, T) .
$$

It follows from (3.7) that

$$
u_{x}^{\epsilon}=\frac{1}{2 \sqrt{\alpha}}(p+q)+\left\|u_{0}\right\|_{L i p^{+}} .
$$


This identity, together with (3.12), yields

$$
u_{x}^{\epsilon} \leq\left\|u_{0}\right\|_{L^{i p^{+}}}
$$

which is the Lip $^{+}$stability (3.3) for $u^{\epsilon}$ when it is smooth. Finally, we extend our result to general initial data by the following standard procedure:

$$
u_{0}^{\delta}(x):=\int \psi_{\delta}(x-y) u_{0}(y) d y
$$

where $\psi_{\delta}$ is a compactly supported nonnegative unit mass mollifier,

$$
\psi_{\delta}(x)=\frac{1}{\delta} \psi\left(\frac{x}{\delta}\right), \quad \int_{-\infty}^{\infty} \psi_{\delta}(x) d x=1 .
$$

It is obvious that if $\left\|u_{0}\right\|_{L i p^{+}}<\infty$, then $\left\|u_{0}^{\delta}\right\|_{L i p^{+}}$is also bounded. Consider the $2 \times 2$ stiff relaxation system (1.1) with the smooth initial data

$$
u^{\epsilon}(x, 0)=u_{0}^{\delta}(x), \quad v^{\epsilon}(x, 0)=f\left(u_{0}^{\delta}(x)\right)
$$

Using the above proof we know that there exists a $T>0$ such that

$$
\left\|u^{\epsilon, \delta}(\bullet, t)\right\|_{L i p^{+}} \leq\left\|u_{0}^{\delta}\right\|_{L i p^{+}} \quad \text { for } \quad t \in(0, T),
$$

where $u^{\epsilon, \delta}$ is one component of the solution to (1.1) and (3.13). Letting $\delta \rightarrow 0+$ in (3.14) gives

$$
\left\|u^{\epsilon}(\bullet, t)\right\|_{L i p^{+}} \leq\left\|u_{0}\right\|_{L^{i p^{+}}} \quad \text { for } \quad t \in(0, T) .
$$

By standard continuation arguments for time, we can extend the desired $\mathrm{Lip}^{+}$stability result (3.3) for $u^{\epsilon}$ to any finite time interval.

We summarize what we have shown by stating the following.

THEOREM 3.1. Assume $\alpha$ in the relaxation equation (1.1) and the initial data $u_{0}$ in (1.2) satisfy the conditions stated in Lemma 2.1. Assume $f^{\prime \prime}>0$. Then the family of solutions $\left\{u^{\epsilon}(x, t)\right\}_{\epsilon>0}$, given by the relaxation system (1.1) and initial data (1.2), are Lip ${ }^{+}$-stable. Moreover, the functions $\left\{\sqrt{\alpha} u^{\epsilon}+v^{\epsilon}\right\}_{\epsilon>0}$ and $\left\{\sqrt{\alpha} u^{\epsilon}-v^{\epsilon}\right\}_{\epsilon>0}$ are also Lip ${ }^{+}$-stable.

3.2. Error estimates based on $\boldsymbol{L i p}^{\prime}$ theory. Equipped with the $\mathrm{Lip}^{+}$-stability, one can derive $\mathrm{O}(\sqrt{\epsilon}) L^{1}$ - and local error bounds using the Lip ${ }^{\prime}$ theory presented in [19]. The case for a general family of relaxation models was outlined in [8]; here is a brief overview for the particular case of the relaxation model (1.1).

To begin with, we derive the modified equation satisfied by $u^{\epsilon}$. Consider the second equation in (1.1),

$$
v_{t}^{\epsilon}+\alpha u_{x}^{\epsilon}=-\frac{1}{\epsilon}\left(v^{\epsilon}-f\left(u^{\epsilon}\right)\right) .
$$

We differentiate with respect to $x$ and use the first equation of (1.1), $v_{x}^{\epsilon}=-u_{t}^{\epsilon}$, to find

$$
u_{t}^{\epsilon}+f\left(u^{\epsilon}\right)_{x}=\epsilon\left(v_{t}^{\epsilon}+\alpha u_{x}^{\epsilon}\right)_{x}
$$


The term on the right is the truncation error. The main result in $[19,14]$ shows that when measured in the $L i p^{\prime}$-norm, the global error, $u^{\epsilon}-u$, is governed by the truncation+initial errors

$$
\left\|u^{\epsilon}-u\right\|_{L i p^{\prime}} \leq \operatorname{Const}\left[\epsilon\left\|\left(v_{t}^{\epsilon}+\alpha u_{x}^{\epsilon}\right)_{x}\right\|_{L i p^{\prime}}+\left\|u_{0}^{\epsilon}-u_{0}\right\|_{L i p^{\prime}}\right] .
$$

In our case of (1.2), there is no initial error. To measure the Lip'-size of the truncation error, we proceed along the lines of [8, Example 3]: we differentiate (1.1) with respect to $t$, obtaining

$$
\begin{aligned}
& \left(u_{t}^{\epsilon}\right)_{t}+\left(v_{t}^{\epsilon}\right)_{x}=0, \\
& \left(v_{t}^{\epsilon}\right)_{t}+\alpha\left(u_{t}^{\epsilon}\right)_{x}=-\frac{1}{\epsilon}\left(v_{t}^{\epsilon}-f^{\prime}\left(u^{\epsilon}\right) u_{t}^{\epsilon}\right) .
\end{aligned}
$$

Performing $\sqrt{\alpha} \times(3.18)+(3.19)$ and $\sqrt{\alpha} \times(3.18)-(3.19)$, then the above system can be put in the following diagonal form in terms of the characteristic variables, $\bar{r}:=\sqrt{\alpha} u_{t}^{\epsilon}+v_{t}^{\epsilon}$ and $\bar{s}:=\sqrt{\alpha} u_{t}^{\epsilon}-v_{t}^{\epsilon}$,

$$
\left\{\begin{array}{l}
\bar{r}_{t}+\sqrt{\alpha} \bar{r}_{x}=\frac{1}{2 \epsilon}\left(\frac{f^{\prime}\left(u^{\epsilon}\right)}{\sqrt{\alpha}}-1\right) \bar{r}+\frac{1}{2 \epsilon}\left(\frac{f^{\prime}\left(u^{\epsilon}\right)}{\sqrt{\alpha}}+1\right) \bar{s}, \\
\bar{s}_{t}-\sqrt{\alpha} \bar{s}_{x}=-\frac{1}{2 \epsilon}\left(\frac{f^{\prime}\left(u^{\epsilon}\right)}{\sqrt{\alpha}}-1\right) \bar{r}-\frac{1}{2 \epsilon}\left(\frac{f^{\prime}\left(u^{\epsilon}\right)}{\sqrt{\alpha}}+1\right) \bar{s} .
\end{array}\right.
$$

Integrate the first equation against $\operatorname{sgn}(\bar{r})$, the second against $\operatorname{sgn}(\bar{s})$, and add; in view of the subcharacteristic condition (2.2) we find (compare [8, equation (4.10)])

$$
\|\bar{r}\|_{L^{1}}+\|\bar{s}\|_{L^{1}} \leq\left\|\bar{r}_{0}\right\|_{L^{1}}+\left\|\bar{s}_{0}\right\|_{L^{1}} .
$$

If the initial data are prepared in the sense that $\left\|v_{0}^{\epsilon}-f\left(u_{0}^{\epsilon}\right)\right\|_{L^{1}}=\mathcal{O}(\epsilon)$ (and in fact, in our case we ignore initial errors by restricting attention to (1.2)), then initial time derivatives

$$
\left\|v_{t}^{\epsilon}(\cdot, t=0)\right\|_{L^{1}}+\left\|u_{t}^{\epsilon}(\cdot, t=0)\right\|_{L^{1}}
$$

are bounded, and by (3.21), they remain bounded in later time. In particular, $\left\|v_{t}^{\epsilon}(\cdot, t)\right\|_{L^{1}} \leq$ Const. This, together with the BV bound of $u^{\epsilon}$ (which follows from the $\mathrm{Lip}^{+}$stability), imply that the $\mathrm{Lip}^{\prime}$-size of the local truncation error is of order $\epsilon$

$$
\left\|\epsilon\left(v_{t}^{\epsilon}+\alpha u_{x}^{\epsilon}\right)_{x}\right\|_{L i p^{\prime}} \leq \epsilon\left(\left\|v_{t}^{\epsilon}\right\|_{L^{1}}+\alpha\left\|u_{x}^{\epsilon}\right\|_{L^{1}}\right) \leq \mathcal{O}(\epsilon),
$$

and consequently, (3.17) implies that the Lip $^{\prime}$ size of the global error, $u^{\epsilon}-u$, is of the same order of $\mathcal{O}(\epsilon)$. If we interpolate between this $\operatorname{Lip}^{\prime}$ bound and the BV boundedness of $u^{\epsilon}-u$, we arrive at an $L^{1}$ convergence rate estimate of order $\mathcal{O}(\sqrt{\epsilon})$,

$$
\left\|u^{\epsilon}-u\right\|_{L^{1}} \leq \text { Const }\left\|u^{\epsilon}-u\right\|_{L i p^{\prime}}^{1 / 2} \cdot\left\|u^{\epsilon}-u\right\|_{B V}^{1 / 2} \leq \text { Const } \sqrt{\epsilon} .
$$

The $\mathrm{Lip}^{+}$stability of $u^{\epsilon}$ enables us to convert this global estimate into a local one: using Lemma 2.3 with $(z, w)=\left(u^{\epsilon}, u\right)$ we find (see (2.10))

$$
\left|u^{\epsilon}(x, t)-u(x, t)\right| \leq \text { Const } \delta \cdot|u|_{C_{l o c}^{1}(x-\delta, x+\delta)}, \quad \delta \sim \epsilon^{1 / 4} .
$$

There are several possible improvements. 
- If one utilizes the $\mathcal{O}(\epsilon)$ - $L i p^{\prime}$ error estimate (instead of the $L^{1}$ estimate of order $\mathcal{O}(\sqrt{\epsilon})$ ), then this pointwise error estimate can be further improved outside a smaller shock region of size $\delta \sim \epsilon^{1 / 3}$ (see [14, Corollary 2.4]).

- Moreover, for piecewise smooth data one has an $L^{1}$-error estimate of order $\epsilon$, [24], and the above arguments yield pointwise error estimate of order $\delta \sim$ $\left\|u^{\epsilon}-u\right\|_{L^{1}}^{1 / 2}=\mathcal{O}(\sqrt{\epsilon})$; this will be outlined in section 3.3.

- Finally, in section 4 we will present a bootstrap argument for a further improvement of this pointwise error estimate; we prove an pointwise error of order $\delta$ outside a shock zone of optimal size $\delta \sim \epsilon$.

Remark. In (1.3) we restrict our attention to initial data which are exactly matched with their assumed limit, $v_{0}^{\epsilon}=f\left(u_{0}^{\epsilon}\right)$. It is clear from the above discussion that Lip $^{\prime}$ error bound of order $\mathcal{O}(\epsilon)$ holds for more general initial data, which are only required to be prepared so that $\left\|u_{0}^{\epsilon}-u_{0}\right\|_{L i p^{\prime}}+\left\|v_{0}^{\epsilon}-f\left(u_{0}^{\epsilon}\right)\right\|_{L^{1}}=\mathcal{O}(\epsilon)$.

3.3. A nonoptimal pointwise error estimate. In the following section, we will consider the case that the entropy solution for (1.3) is piecewise smooth, with finitely many shock discontinuities. Thus, if we let $S(t)$ denote the singular support of $u(\bullet, t)$, then it consists of finitely many shocks, $S(t):=\left\{(x, t) \mid x=X_{k}(t)\right\}$, each of which satisfies the Rankine-Hugoniot and the Lax conditions:

$$
\begin{aligned}
& X_{k}^{\prime}=\frac{\left[f\left(u\left(X_{k}, t\right)\right]\right.}{\left[u\left(X_{k}, t\right)\right]}, \\
& f^{\prime}\left(u\left(X_{k}(t)-, t\right)\right)>X_{k}^{\prime}(t)>f^{\prime}\left(u\left(X_{k}(t)+, t\right)\right) .
\end{aligned}
$$

We note in passing that many practical initial data lead to finite number of shocks (see, e.g., [17, 22]), and in this case one has a global $L^{1}$-error bound of order $\epsilon,(2.7)$. Next we consider the characteristic variables, $\sqrt{\alpha} u^{\epsilon} \pm v^{\epsilon}$ : It follows that their $L^{1}$ convergence rate from their limiting value $\sqrt{\alpha} u \pm v$ with $v=f(u)$ is also order $(\epsilon)$. Moreover, Theorem 3.1 implies the Lip $^{+}$boundedness

$$
\sqrt{\alpha} u_{x}^{\epsilon}+v_{x}^{\epsilon} \leq C, \quad \sqrt{\alpha} u_{x}^{\epsilon}-v_{x}^{\epsilon} \leq C .
$$

We can now apply the interpolation inequality (2.10), with $(z, w)=\left(\sqrt{\alpha} u^{\epsilon} \pm v^{\epsilon}, \sqrt{\alpha} u \pm\right.$ $f(u)$ ). We obtain the following pointwise error bound (see also [20]):

$$
\left\{\begin{array}{l}
\left|\sqrt{\alpha} u^{\epsilon}+v^{\epsilon}-(\sqrt{\alpha} u+f(u))\right| \leq C \sqrt{\epsilon} \\
\left|\sqrt{\alpha} u^{\epsilon}-v^{\epsilon}-(\sqrt{\alpha} u-f(u))\right| \leq C \sqrt{\epsilon} \quad \text { for } \quad \operatorname{dist}(x, S(t)) \geq \sqrt{\epsilon}
\end{array}\right.
$$

It follows from the above results that

$$
\left\{\begin{array}{l}
\left|u^{\epsilon}(x, t)-u(x, t)\right| \leq C \sqrt{\epsilon} \\
\left|v^{\epsilon}(x, t)-f(u(x, t))\right| \leq C \sqrt{\epsilon} \quad \text { for } \quad \operatorname{dist}(x, S(t)) \geq \sqrt{\epsilon} .
\end{array}\right.
$$

Although the above pointwise local estimate is not optimal, it will suffice to derive the optimal error bound by a bootstrap argument which employs the comparison Lemma 2.4.

4. Pointwise error estimate. The key tool in obtaining the optimal pointwise error estimate is to use Lemma 2.4. In order to use it, we need to construct appropriate functions $u_{1}$ and $u_{2}$ (in this section they are error functions) such that they satisfy (2.11) and those conditions listed in the lemma. To illustrate the main idea of our proof, we first concentrate on the case that there is only one shock curve. 
4.1. The case of a single shock. We assume that there is a smooth curve, $S(t):=\{(x, t) \mid x=X(t)\}$, so that $u(x, t)$ is $C^{2}$-smooth at any point $x \neq X(t)$. There are two smooth regions $x>X(t)$ and $x<X(t)$. We consider the pointwise error estimate in the region $x>X(t)$; the results for $x<X(t)$ can be obtained in a similar way. The function $\phi(x) \in C^{2}([0, \infty))$ satisfies

$$
\phi(x) \sim\left\{\begin{array}{lll}
x & \text { if } & 0 \leq x \ll 1 \\
1 & \text { if } & x \gg 1
\end{array}\right.
$$

More precisely, the function $\phi$ satisfies

$$
\left\{\begin{array}{l}
\phi(0)=0, \quad \phi^{\prime}(x)>0, \quad \phi(x) \leq x \quad \text { for } \quad x>0 \\
x \phi^{\prime}(x) \leq \phi(x) \quad \text { for } \quad x \geq 0 \\
\left|\phi^{(k)}(x)\right| \leq 1, \quad x \geq 0
\end{array}\right.
$$

e.g., $\phi(x)=1-e^{-x}$. Roughly speaking, the weighted function behaves like $\phi(x) \sim$ $\min (|x|, 1)$.

We define two functions, which roughly speaking are the errors for $u^{\epsilon}$ and $v^{\epsilon}$, in the following form:

$$
U=u^{\epsilon}-u-\sigma(x, t), \quad V=v^{\epsilon}-f(u+\sigma)+\epsilon \Psi(x, t),
$$

where

$$
\left\{\begin{array}{l}
\sigma=\epsilon d e^{\gamma t} / \phi(x-X(t)) \\
\Psi=\left(\alpha-f^{\prime}(u+\sigma) f^{\prime}(u)\right) u_{x}-\left(\sqrt{\alpha}+f^{\prime}(u+\sigma)\right)(\sqrt{\alpha}-\dot{X}(t)) \sigma \phi^{\prime} / \phi
\end{array}\right.
$$

In the above definitions, $\phi=\phi(x-X(t))$ is the so-called weighted distance to the shock set. ${ }^{2}$ Also, in the above definitions, $d$ and $\gamma$ are two positive numbers to be determined.

Remark. It is seen from (4.2) and (4.3) that $U$ is the error function for $u^{\epsilon}$ with first-order correction $O(\epsilon)$, while $V$ is the error function for $v^{\epsilon}$ with first- and secondorder corrections.

4.1.1. The basic idea. In order to put the error functions $U$ and $V$ to the framework of Lemma 2.4, we further let

$$
p=\sqrt{\alpha} U+V, \quad q=\sqrt{\alpha} U-V
$$

and will verify the following estimates:

- (C1): for $x \geq X(0)+\sqrt{\epsilon}$,

$$
p(x, 0) \leq 0, \quad q(x, 0) \leq 0 .
$$

- (C2): for all $t \geq 0$,

$$
p(X(t)+\sqrt{\epsilon}, t) \leq 0, \quad q(X(t)+\sqrt{\epsilon}, t) \leq 0 .
$$

\footnotetext{
${ }^{2}$ In the case $x<X(t)$, the weighted distance is $\phi(X(t)-x)$. In other words, the weighted distance for any choice of $x$ is $\phi(|x-X(t)|)$ in the single shock case.
} 
- (C3): The functions $p$ and $q$ satisfies the following equations:

$$
\left\{\begin{array}{l}
p_{t}+\sqrt{\alpha} p_{x}=\alpha_{11} p+\alpha_{12} q+\beta_{1}(x, t), \\
q_{t}-\sqrt{\alpha} q_{x}=\alpha_{21} p+\alpha_{22} q+\beta_{2}(x, t),
\end{array}\right.
$$

where for $x \geq X(t)+\sqrt{\epsilon}$ the coefficients $\alpha_{12}$ and $\alpha_{21}$ are nonnegative, and the source terms $\beta_{1}$ and $\beta_{2}$ are nonpositive.

The idea is to choose $d$ and $\gamma$ sufficiently large so that Lemma 2.4 can be applied. The estimates $(\mathrm{C} 1)$ and $(\mathrm{C} 2)$ are satisfied by choosing sufficiently large $d$. Then for the time interval $0<t \leq T_{1}:=\gamma^{-1}$, i.e.,

$$
e^{\gamma t} \leq e
$$

we show that (C3) is satisfied by choosing sufficiently large $\gamma$.

After showing that $(\mathrm{C} 1)-(\mathrm{C} 3)$ are verified for $t \in\left[0, T_{1}\right]$, we know that the error bounds for $u^{\epsilon}$ and $v^{\epsilon}$ can be established for $0 \leq t \leq T_{1}$. We can then use $u^{\epsilon}\left(x, T_{1}\right)$ and $u\left(x, T_{1}\right)$ as new initial data and repeat the same procedure to obtain the local error bounds for $T_{1}<t \leq T_{2}$. By this standard continuation arguments, we can obtain the error bounds up to $t=T$.

4.1.2. The verification for (C1). Observe that for $x \geq X(t)+\sqrt{\epsilon}$

$$
|\Psi(x, t)| \leq C+C \epsilon^{-1 / 2} \sigma, \quad \sigma \geq \epsilon d .
$$

Since $u^{\epsilon}(x, 0)=u(x, 0)=u_{0}(x)$ and $v^{\epsilon}(x, 0)=f\left(u_{0}\right)$, we have, for $x \geq X(0)+\geq \sqrt{\epsilon}$,

$$
\begin{aligned}
p(x, 0) & =-\sqrt{\alpha} \sigma(x, 0)+f\left(u_{0}\right)-f\left(u_{0}+\sigma\right)+\epsilon \Psi \\
& =\left(-\sqrt{\alpha}+f^{\prime}(\bullet)\right) \sigma(x, 0)+\epsilon \Psi \\
& \leq-C_{1} \sigma+C \epsilon+C \sqrt{\epsilon} \sigma \\
& \leq\left(-C_{1}+C / d+C \sqrt{\epsilon}\right) \sigma \leq 0
\end{aligned}
$$

provided that $d$ is sufficiently large and $\epsilon$ sufficiently small. Similarly, we can show that $q(x, 0)<0$ for $x>X(0)+\sqrt{\epsilon}$ with sufficiently large $d$ and small $\epsilon$.

4.1.3. The verification for (C2). It follows from the nonoptimized local error estimates (3.27) that

$$
u^{\epsilon}-u=\mathcal{O}(\sqrt{\epsilon}), \quad v^{\epsilon}-f(u)=\mathcal{O}(\sqrt{\epsilon}) \quad \text { for } \quad x \geq X(t)+\sqrt{\epsilon} .
$$

It is also observed that $\sigma(x, t) \geq C d \sqrt{\epsilon}$ for $x=X(t)+\sqrt{\epsilon}$. From the definition of $p$ we obtain

$$
\begin{aligned}
p(X(t)+\sqrt{\epsilon}, t) & =\sqrt{\alpha} \times \mathcal{O}(\sqrt{\epsilon})-\sqrt{\alpha} \sigma(x, t)+\mathcal{O}(\sqrt{\epsilon})+f(u)-f(u+\sigma)+\mathcal{O}(\epsilon)+\mathcal{O}(\epsilon \sigma) \\
& =\mathcal{O}(\sqrt{\epsilon})+\left(-\sqrt{\alpha}-f^{\prime}(\bullet)\right) \sigma(X(t)+\sqrt{\epsilon}, t)+\mathcal{O}(\epsilon)+\mathcal{O}(\epsilon \sigma) \\
& \leq C \sqrt{\epsilon}-C_{1} C \sqrt{\epsilon} d+C \epsilon \leq 0
\end{aligned}
$$

provided that $d$ is sufficiently large. Similarly, we can show that $q(X(t)+\sqrt{\epsilon}, t) \leq 0$. 
4.1.4. The verification for (C3). By the definitions of $U$ and $V$, as well as the relaxation equations (1.1) and its equilibrium equation (1.3), we have

$$
\begin{aligned}
U_{t}+V_{x} & =\underbrace{u_{t}^{\epsilon}+v_{x}^{\epsilon}}_{=0}-\underbrace{\left(u_{t}+f(u)_{x}\right)}_{=0}+\left(f(u)_{x}-f(u+\sigma)_{x}\right)-\sigma_{t}+\epsilon \Psi_{x} \\
& =\left(f^{\prime}(u)-f^{\prime}(u+\sigma)\right) u_{x}-f^{\prime}(u+\sigma) \sigma_{x}-\sigma_{t}+\epsilon \Psi_{x} \\
& =-f^{\prime \prime}(\bullet) u_{x} \sigma-f^{\prime}(u+\sigma) \sigma_{x}-\sigma_{t}+\epsilon \Psi_{x} .
\end{aligned}
$$

Similarly, we calculate $V_{t}+\alpha U_{x}$ and obtain

$$
\begin{gathered}
V_{t}+\alpha U_{x}=v_{t}^{\epsilon}+\alpha u_{x}^{\epsilon}-f(u+\sigma)_{t}-\alpha u_{x}+\epsilon \Psi_{t}-\alpha \sigma_{x} \\
=\frac{1}{\epsilon}\left(f\left(u^{\epsilon}\right)-v^{\epsilon}\right)-f^{\prime}(u+\sigma)\left(u_{t}+\sigma_{t}\right)-\alpha u_{x}+\epsilon \Psi_{t}-\alpha \sigma_{x} \\
=\frac{1}{\epsilon}\left(f\left(u^{\epsilon}\right)-f(u+\sigma)\right)-\frac{1}{\epsilon}\left(v^{\epsilon}-f(u+\sigma)+\epsilon \Psi\right) \\
\quad-f^{\prime}(u+\sigma) \sigma_{t}+\epsilon \Psi_{t}-\alpha \sigma_{x}+\Psi+\left(f^{\prime}(u+\sigma) f^{\prime}(u)-\alpha\right) u_{x} \\
=\frac{1}{\epsilon} f^{\prime} \bullet \bullet-\frac{1}{\epsilon} V-f^{\prime}(u+\sigma) \sigma_{t}+\epsilon \Psi_{t}-\alpha \sigma_{x} \\
\quad-\left(\sqrt{\alpha}+f^{\prime}(u+\sigma)\right)(\sqrt{\alpha}-\dot{X}(t)) \frac{\sigma \phi^{\prime}}{\phi} .
\end{gathered}
$$

By the definition of $p, p=\sqrt{\alpha} U+V$, we obtain from (4.8) and (4.9) that $p$ satisfies the first equation in (4.5) with

$$
\left\{\begin{array}{l}
\alpha_{11}=\frac{1}{2 \epsilon}\left(\frac{f^{\prime}(\bullet)}{\sqrt{\alpha}}-1\right), \quad \alpha_{12}=\frac{1}{2 \epsilon}\left(\frac{f^{\prime}(\bullet)}{\sqrt{\alpha}}+1\right) \\
\beta_{1}(x, t)=-\sqrt{\alpha} f^{\prime \prime}(\bullet) u_{x} \sigma-\left(\sqrt{\alpha}+f^{\prime}(u+\sigma)\right)\left(\sqrt{\alpha} \sigma_{x}+\sigma_{t}\right) \\
+\epsilon\left(\sqrt{\alpha} \Psi_{x}+\Psi_{t}\right)-\left(\sqrt{\alpha}+f^{\prime}(u+\sigma)\right)(\sqrt{\alpha}-\dot{X}(t)) \frac{\sigma \phi^{\prime}}{\phi} .
\end{array}\right.
$$

We observe that $\alpha_{12} \geq 0$. Now we need to verify that $\beta_{1}(x, t) \leq 0$ for $x \geq X(t)+\sqrt{\epsilon}$ provided that $\gamma$ is sufficiently large. It follows from the definitions of $\sigma$ that

$$
\sigma_{x}=-\frac{\sigma \phi^{\prime}}{\phi}, \quad \sigma_{t}=\gamma \sigma+\frac{\sigma \phi^{\prime}}{\phi} \dot{X} .
$$

Using the above results and the definition of $\Psi$ gives

$$
\begin{aligned}
\Psi_{x} & =\mathcal{O}(1)+\mathcal{O}\left(\frac{\sigma^{2}}{\phi^{2}}\right)+\mathcal{O}\left(\frac{\sigma}{\phi^{2}}\right) \\
& \leq C+C \epsilon^{-1} \sigma^{2}+C \epsilon^{-1} \sigma \leq C+C \epsilon^{-1} \sigma \quad(\text { using (4.6)) } \\
\Psi_{t} & =\mathcal{O}(1)+\mathcal{O}\left(\frac{\sigma^{2}}{\phi^{2}}\right)+\mathcal{O}\left(\frac{\sigma}{\phi^{2}}\right)+\mathcal{O}\left(\frac{\sigma^{2}}{\phi}\right) \\
& \leq C+C \epsilon^{-1} \sigma+C \sigma .
\end{aligned}
$$

The above estimates, together with (4.11) and the definition of $\beta_{1}(x, t)$, yield

$$
\begin{aligned}
\beta_{1}(x, t) & =-\sqrt{\alpha} f^{\prime \prime}(\bullet) u_{x} \sigma-\left(\sqrt{\alpha}+f^{\prime}(u+\sigma)\right) \gamma \sigma+\epsilon\left(\sqrt{\alpha} \Psi_{x}+\Psi_{t}\right) \\
& \leq C \sigma-C_{1} \gamma \sigma+C \epsilon .
\end{aligned}
$$


From the definition of $\psi$ we have $\epsilon=\phi e^{-\gamma t} \sigma / d \leq C \sigma / d$. This, together with (4.12), gives

$$
\beta_{1}(x, t) \leq\left(C-C_{1} \gamma+C / d\right) \sigma \leq 0 \quad \text { for } \quad x \geq X(t)+\sqrt{\epsilon}
$$

provided that $\gamma$ is sufficiently large.

It follows from (4.8)-(4.9) and the definition of $q, q \sqrt{\alpha} U-V$, that $q$ satisfies the second equation in (4.5) with

$$
\left\{\begin{aligned}
\alpha_{21}=\frac{1}{2 \epsilon} & \left(1-\frac{f^{\prime}(\bullet)}{\sqrt{\alpha}}\right), \quad \alpha_{22}=-\frac{1}{2 \epsilon}\left(1+\frac{f^{\prime}(\bullet)}{\sqrt{\alpha}}\right) \\
\beta_{2}(x, t)= & -\sqrt{\alpha} f^{\prime \prime}(\bullet) u_{x} \sigma+\left(\sqrt{\alpha}-f^{\prime}(u+\sigma)\right)\left(\sqrt{\alpha} \sigma_{x}-\sigma_{t}\right)+\epsilon\left(\sqrt{\alpha} \Psi_{x}-\Psi_{t}\right) \\
& +\left(\sqrt{\alpha}+f^{\prime}(u+\sigma)\right)(\sqrt{\alpha}-\dot{X}) \frac{\sigma \phi^{\prime}}{\phi}
\end{aligned}\right.
$$

It is seen that $\alpha_{21} \geq 0$. Moreover, it follows from (4.11) that

$$
\beta_{2}(x, t)=-\sqrt{\alpha} f^{\prime \prime}(\bullet) u_{x} \sigma-\left(\sqrt{\alpha}-f^{\prime}(u+\sigma)\right) \gamma \sigma+\epsilon\left(\sqrt{\alpha} \Psi_{x}+\Psi_{t}\right)+J
$$

where the last term $J$ is defined by

$$
J=2 \sqrt{\alpha}\left(f^{\prime}(u+\sigma)-\dot{X}\right) \frac{\sigma \phi^{\prime}}{\phi} .
$$

Let $u_{+}:=u(X(t)+0, t)$. Using Lax geometrical entropy condition $\dot{X}(t) \geq f^{\prime}\left(u_{+}\right)$ gives

$$
\begin{aligned}
& f^{\prime}(u+\sigma)-\dot{X}(t) \leq f^{\prime}(u+\sigma)-f^{\prime}\left(u_{+}\right) \\
= & f^{\prime \prime}(\bullet)(u(x, t)-u(X(t)+0, t))+f^{\prime \prime}(\bullet) \sigma \\
= & f^{\prime \prime}(\bullet) u_{x}(\bullet, t)(x-X(t))+f^{\prime \prime}(\bullet) \sigma .
\end{aligned}
$$

Using the fact that $x \phi^{\prime}(x) \leq \phi(x)$ gives

$$
(x-X(t)) \phi^{\prime}(x-X(t)) \leq C \phi(x-X(t)) .
$$

By the definition of $J$ and the above observations, we have

$$
J \leq C \sigma+C \frac{\sigma^{2}}{\phi} \leq C \sigma,
$$

where in the last step we have used the fact $\sigma / \phi \leq C d$. It follows from the above results and the equation for $\beta_{2}$, (4.15), that $\beta_{2} \leq 0$ provided that $\gamma$ is sufficiently large.

In summary, if $d$ and $\gamma=\gamma(d)$ are sufficiently large, then the comparison lemma, Lemma 2.4, gives

$$
p(x, t) \leq 0, \quad q(x, t) \leq 0 \quad \text { for } \quad x \geq X(t)+\sqrt{\epsilon} .
$$

Similarly, changing $\phi(x-X(t))$ in (4.3) to $\phi(X(t)-x)$ will handle the case for $x \leq$ $X(t)-\sqrt{\epsilon}$. We will then obtain the following results:

$$
p(x, t) \leq 0, \quad q(x, t) \leq 0 \quad \text { for } \quad x \leq X(t)-\sqrt{\epsilon} .
$$


Since

$$
u^{\epsilon}-u-\epsilon d e^{\gamma t} / \phi=\frac{1}{2 \sqrt{\alpha}}(p+q),
$$

the estimates (4.17) and (4.18) yield

$$
u^{\epsilon}-u \leq \epsilon d e^{\gamma t} / \phi \quad \text { for } \quad|x-X(t)| \geq \sqrt{\epsilon} .
$$

By letting $U=u-u^{\epsilon}-\sigma$ and $V=f(u-\sigma)-v^{\epsilon}+\epsilon \tilde{\Psi}$, where $\tilde{\Psi}$ is of a similar form for $\Psi$, we can again using the comparison lemma obtain

$$
u-u^{\epsilon} \leq \epsilon d e^{\gamma t} / \phi \quad \text { for } \quad|x-X(t)| \geq \sqrt{\epsilon} .
$$

We summarize what we have shown by stating the following.

ASSERTION 4.1. Let $u^{\epsilon}(x, t)$ be the relaxation solutions of (1.1)-(1.2) and $u(x, t)$ be the entropy solution of (1.3). If the entropy solution has only one shock discontinuity $S(t)=\{(x, t) \mid x=X(t)\}$, then the following error estimates hold:

- For a weighted distance function $\phi, \phi(x) \sim \min (|x|, 1)$,

$$
\left|\left(u^{\epsilon}-u\right)(x, t)\right| \phi(|x-X(t)|)=\mathcal{O}(\epsilon), \quad|x-X(t)| \geq \sqrt{\epsilon} .
$$

- In particular, if $(x, t)$ is away from the singular support, then

$$
\left|\left(u^{\epsilon}-u\right)(x, t)\right| \leq C(h) \epsilon, \quad \operatorname{dist}(x, S(t)) \geq h .
$$

4.2. Finitely many shocks. In the case that the entropy solutions for the conservation law (1.3) have two shocks, our analysis in section 4.1 can be extended to cover this case easily. The main difference is to change the weighted distance function $\phi$ to the product of two weighted distance functions, i.e., $\phi\left(\left|x-X_{1}(t)\right|\right) \cdot \phi\left(\left|x-X_{2}(t)\right|\right)$. This idea was used in [20].

In a more general case when there are finitely many shocks, we replace the weighted distance function by

$$
\rho(x, t)=\prod_{k=1}^{K} \phi\left(\left|x-X_{k}(t)\right|\right) .
$$

Then we consider the error functions similar to (4.3). We can apply the same techniques as used in the last subsection to obtain the optimal error bounds. We omit the detail procedure but state our main result as follows.

THEOREM 4.1. Let $u^{\epsilon}(x, t)$ be the relaxation solutions of $(1.1)-(1.2)$ and $u(x, t)$ be the entropy solution of (1.3). If the entropy solution has finitely many shock discontinuities, $S(t)=\left\{(x, t) \mid x=X_{k}(t)\right\}_{k=1}^{K}$, then the following error estimates hold:

- For a weighted distance function $\phi, \phi(x) \sim \min (|x|, 1)$,

$$
\left|\left(u^{\epsilon}-u\right)(x, t)\right| \prod_{k=1}^{K} \phi\left(\left|x-X_{k}(t)\right|\right)=\mathcal{O}(\epsilon), \quad|x-X(t)| \geq \sqrt{\epsilon}
$$

- In particular, if $(x, t)$ is away from the singular support, then

$$
\left|\left(u^{\epsilon}-u\right)(x, t)\right| \leq C(h) \epsilon, \quad \operatorname{dist}(x, S(t)) \geq h .
$$

It is noted that the above results cover the case when there are finitely many shocks with possible collisions. 
5. Concluding remarks. In this work, we have obtained the pointwise error bounds for relaxation approximations to scalar conservation laws with piecewise smooth solutions. The proof of our results is based on two ingredients: a onesided interpolation inequality (interpolating the $L^{1}$ error estimates and Lip ${ }^{+}$stability bounds), and a comparison theorem for weakly coupled hyperbolic systems. Here, we only investigated the case of entropy solutions of the equilibrium equation (1.3) which consist of finitely many shocks. The techniques used in this paper can be extended, however, in several directions:

- Finitely many rarefaction waves. Combining the techniques presented in [20], sharp pointwise error bounds can be obtained for entropy solutions of the equilibrium equation (1.3) which consist of finitely many rarefactions.

- Finite difference approximations. Sharp pointwise error bounds can be obtained for difference approximations of the equilibrium equation (1.3). A convergence study based on Lip' arguments was presented in [14]. Augmented with one-sided interpolation together with appropriate comparison techniques along the lines of our discussion in section 4 , one can convert the global $\mathrm{Lip}^{\prime}$ error estimates into sharp pointwise error estimates. The example of Lax-Friedrichs central scheme, corresponding to the first-order relaxation scheme of [6], was worked out by the authors in [21]. The second-order schemes based on the relaxation approximation (1.1) correspond to the central scheme in [13], and like most high-resolution schemes, the main difficulty lies with the question of their $\mathrm{Lip}^{+}$stability.

\section{REFERENCES}

[1] G.-Q. Chen, C. Levermore, And T. P. LiU, Hyperbolic conservation laws with stiff relaxation terms and entropy, Comm. Pure Appl. Math., 47 (1994), pp. 787-830.

[2] B. Engquist And B. SJogreEn, The convergence rate of finite difference schemes in the presence of shocks, SIAM J. Numer. Anal., 35 (1998), pp. 2464-2485.

[3] B. Engquist and S.-H. Yu, Convergence of Finite Difference Schemes for Piecewise Smooth Solutions with Shocks, preprint, 1997.

[4] J. Goodman And Z. XIN, Viscous limits for piecewise smooth solutions to systems of conservation laws, Arch. Rational Mech. Anal., 121 (1992), pp. 235-265.

[5] G.-S. JiAng AND E. TADMOR, Nonoscillatory central schemes for multidimensional hyperbolic conservation laws, SIAM J. Sci. Comput., 19 (1998), pp. 1892-1917.

[6] S. JIN AND Z. XIN, The relaxation schemes for system of conservation laws in arbitrary space dimensions, Comm. Pure Appl. Math., 48 (1995), pp. 235-277.

[7] M. A. Katsoulakis and A. E. Tzavaras, Contractive relaxation systems and the scalar multidimensional conservation law, Comm. Partial Differential Equations, 22 (1997), pp. 195233.

[8] A. Kurganov And E. Tadmor, Stiff systems of hyperbolic conservation laws: Convergence and error estimates, SIAM J. Math. Anal., 28 (1997), pp. 1446-1456.

[9] N. N. Kuznetsov, Accuracy of some approximate methods for computing the weak solutions of a first-order quasi-linear equation, U.S.S.R. Comput. Math. and Math. Phys., 16 (1976), pp. $105-119$.

[10] P. L. Lions, B. Perthame, and E. Tadmor, Kinetic formulation of scalar conservation laws, J. Amer. Math. Soc., 7 (1994), pp. 169-191.

[11] T. P. LIU, Hyperbolic conservation laws with relaxation, Comm. Math. Phys., 108 (1987), pp. $153-175$.

[12] R. NATALini, Convergence to equilibrium for the relaxation approximations of conservation laws, Comm. Pure Appl. Math., 49 (1996), pp. 795-823.

[13] H. Nessyahu and E. Tadmor, Non-oscillatory central differencing for hyperbolic conservation laws, J. Comput. Phys., 87 (1990), pp. 408-463.

[14] H. Nessyahu And E. TADMOR, The convergence rate of approximate solutions for nonlinear scalar conservation laws, SIAM J. Numer. Anal., 29 (1992), pp. 1505-1519. 
[15] B. Perthame and E. Tadmor, A kinetic equation with kinetic entropy functions for scalar conservation laws, Comm. Math. Phys., 136 (1991), pp. 501-517.

[16] M. H. Protter and H. F. Weinberger, Maximum Principles in Differential Equations, Prentice-Hall, Englewood Cliffs, NJ, 1967.

[17] D. G. Schaeffer, A regularity theorem for conservation laws, Adv. Math., 11 (1973), pp. 368386.

[18] H. J. Schroll, A. Tveito, And R. Winther, An $L^{1}$-error bound for a semi-implicit difference scheme applied to a stiff system of conservation laws, SIAM J. Numer. Anal., 34 (1997), pp. $1152-1166$.

[19] E. TADMOR, Local error estimates for discontinuous solutions of nonlinear hyperbolic equations, SIAM J. Numer. Anal., 28 (1991), pp. 891-906.

[20] E. TAdmor And T. TANG, Pointwise error estimates for scalar conservation laws with piecewise smooth solutions, SIAM J. Numer. Anal., 36 (1999), pp. 1739-1758.

[21] E. TAdmor and T. TAng, Pointwise convergence rate for nonlinear conservation laws, in Hyperbolic Problems: Theory, Numerics, Applications, M. Fey and R. Jeltsch, eds., Internat. Ser. Numer. Math. 130, Birkhäuser, Basel, 1999, pp. 925-934.

[22] E. TAdmor and T. TASSA, On the piecewise smoothness of entropy solutions to scalar conservation laws, Comm. Partial Differential Equations, 18 (1993), pp. 1631-1652.

[23] T. TANG AND Z.-H. Teng, Viscosity methods for piecewise smooth solutions to scalar conservation laws, Math. Comp., 66 (1997), pp. 495-526.

[24] Z. H. TENG, First-order $L^{1}$-convergence for relaxation approximations to conservation laws, Comm. Pure Appl. Math., 51 (1998), pp. 857-895.

[25] Z.-H. Teng AND P. Zhang, Optimal $L^{1}$-rate of convergence for the viscosity method and monotone scheme to piecewise constant solutions with shocks, SIAM J. Numer. Anal., 34 (1997), pp. 959-978.

[26] A. TVeito and R. Winther, On the rate of convergence to equilibrium for a system of conservation laws including a relaxation term, SIAM J. Math. Anal., 28 (1997), pp. 136-161.

[27] W.-C. Wang And Z. XIN, Asymptotic limit of initial boundary value problems for conservation laws with relaxation extensions, Comm. Pure Appl. Math., 51 (1998), pp. 505-535.

[28] G. Whitham, Linear and Nonlinear Waves, Wiley-Interscience, New York, 1974. 